\title{
Blue InGaN/GaN-based \\ Quantum Electroabsorption Modulators
}

\author{
Emre Sari*ł, Sedat Nizamoglu ${ }^{\dagger \ddagger}$, Tuncay Ozel ${ }^{\dagger \ddagger}$, and Hilmi Volkan Demir ${ }^{* 1 \neq}$ \\ *Department of Electrical and Electronics Engineering, ${ }^{\dagger}$ Department of Physics, ${ }^{\dagger}$ Nanotechnology Research Center \\ Bilkent University, Ankara, Turkey TR-06800 \\ Email: volkan@bilkent.edu.tr, Telephone: [+90](312) 290-1021, Fax: [+90](312) 290-1015
}

\begin{abstract}
We introduce InGaN/GaN-based quantum electroabsorption modulator that incorporates $-5 \mathrm{~nm}$ thick $\mathrm{In}_{0.35} \mathrm{Ga}_{0.65} \mathrm{~N} / \mathrm{GaN}$ quantum structures for operation in the blue spectral range of $420-430 \mathrm{~nm}$. This device exhibits an optical absorption coefficient change of $\sim 6000 \mathrm{~cm}^{-1}$ below the band edge at highly transmissive, blue region (at $\lambda_{\text {peak }}=424 \mathrm{~nm}$ ) with a $6 \mathrm{~V}$ swing and emits blue light (at $\lambda_{\text {peak }}=440 \mathrm{~nm}$ ) with an optical output power of $0.35 \mathrm{~mW}$ at a $20 \mathrm{~mA}$ current injection level. Unlike infrared III-V quantum modulators, this blue modulator shows a blue shift in its electroabsorption (for $\lambda<\mathbf{4 1 8} \mathbf{~ n m}$ ) with increasing applied field accross it, due to high alternating polarization fields in its quantum structures; this electroabsorption behavior is opposite to the conventional quantum confined Stark effect that features common red shift. This device holds great promise for $>10 \mathrm{GHz}$ optical clock injection directly into silicon CMOS chips in the blue because of its low parasitic in-series resistance $(<100 \Omega)$ and the possibility to make smaller device mesas for low capacitance $(1.2 \mathrm{fF}$ for a $10 \mu \mathrm{m} \times 10 \mu \mathrm{m}$ mesa size). Considering high-speed operation and high responsivity of silicon-on-insulator (SOI) photodetectors in the blue range, unlike in the infrared, this approach eliminates the need for on-chip hybrid integration of Si CMOS with III-V photodetectors. Furthermore, the efficient electroluminescence of this device makes it feasible to consider on-chip blue lasermodulator integration for a compact optical clocking scheme.
\end{abstract}

Keywords-modulator; electroabsorption; quantum structure; GaN, InGaN.

\section{INTRODUCTION}

Silicon is a good material for photodetection of the blue light due to its short absorption depth in the blue $(\sim 100 \mathrm{~nm}$ at $\lambda=400 \mathrm{~nm}$ ). Therefore, Si photodetectors fabricated in standard CMOS process favorably lack the diffusion tail problem when detecting the blue light, unlike the infrared. Thus, optical clock injection in this region of the optical spectrum enables highspeed optical interconnects and clock injection directly into Si CMOS (e.g. at $>10 \mathrm{Gbps}$ ), without having to use a hybrid integrated compound semiconductor detector on CMOS [1]. The recent advances in GaN optoelectronics technology have produced high-brightness light emitting diodes and laser diodes [2] across the entire visible spectrum down to the ultraviolet.

To utilize the technological progress in $\mathrm{GaN}$ growth and processing and to address the demand for optical clock injection directly into CMOS, we develop blue InGaN/GaN- based quantum electroabsorption modulators; here we present their epitaxial growth, fabrication and experimental characterization.

\section{GROWTH AND FABRICATION}

The architecture of our modulators is based on a surfacenormal $p-i-n$ structure that houses InGaN/GaN quantum structures in its intrinsic region. Our epitaxial wafers are grown on c-plane double side polished sapphire substrates using AIXTRON RF200/4 RF-S metal organic chemical vapor deposition (MOCVD) system located at Bilkent University Nanotechnology Research Center. In our MOCVD, TEGa (for quantum structures), TMGa (for bulk layers), TMIn, TMAl and $\mathrm{NH}_{3}$ are used as precursors.

The epitaxial growth is initiated with a $14 \mathrm{~nm}$ thick GaN nucleation layer and a $200 \mathrm{~nm}$ thick GaN buffer layer, and is followed by a $690 \mathrm{~nm}$ thick Si doped $\mathrm{GaN}$ layer (n-type contact layer) and subsequently five $\sim 5 \mathrm{~nm}$ thick $\operatorname{In}_{0.35} \mathrm{Ga}_{0.65} \mathrm{~N}$ quantum well and $\sim 5 \mathrm{~nm}$ thick GaN barrier structures grown at $682^{\circ} \mathrm{C}$, and is finalized with $\mathrm{Mg}$ doped $50 \mathrm{~nm}$ thick $\mathrm{Al}_{0.1} \mathrm{Ga}_{0.9} \mathrm{~N}$ and $120 \mathrm{~nm}$ thick GaN layers-both p-type, the latter being the contact cap layer.

We start the fabrication with the dehydrogenation of $\mathrm{Mg}$ dopants by annealing our epitaxial wafers at $750^{\circ} \mathrm{C}$ for 15 minutes under $\mathrm{N}_{2}$ purge. We use standart lithography for reactive ion etching of device mesas and subsequent metallization steps. We evaporate $\mathrm{Ni}: \mathrm{Au}$ and Ti:Al for $\mathrm{p}$ - and n-contacts, respectively, both being $10 / 100 \mathrm{~nm}$ thick. We finally apply rapid thermal annealing at $650^{\circ} \mathrm{C}$ for 1 minute. Our fabricated devices have mesa sizes varying from $10 \mu \mathrm{m} \times 10 \mu \mathrm{m}$ (corresponding to $1.2 \mathrm{fF}$ ) to $300 \mu \mathrm{mx} 300 \mu \mathrm{m}$ (corresponding to $1.5 \mathrm{pF}$ ), with open optical windows to increase incident light coupling into the device in operation.

\section{ChaRACTERIZATION}

We perform photoluminescence (PL) characterization at room temperature using a He-Cd laser as the excitation source at an excitation wavelength of $325 \mathrm{~nm}$. We observe the PL peak to be at $430 \mathrm{~nm}$ as shown in Fig. 1a. This spectrum verifies that the quantum wells are made of $\operatorname{In}_{0.35} \mathrm{Ga}_{0.65} \mathrm{~N}$. Figure $1 \mathrm{~b}$ shows the electroluminescence spectrum (EL) of the device for a driving current of $10 \mathrm{~mA}$. The total optical power 
is $0.35 \mathrm{~mW}$ at a $20 \mathrm{~mA}$ injection current level and the $\mathrm{EL}$ peak wavelength is $440 \mathrm{~nm}$.
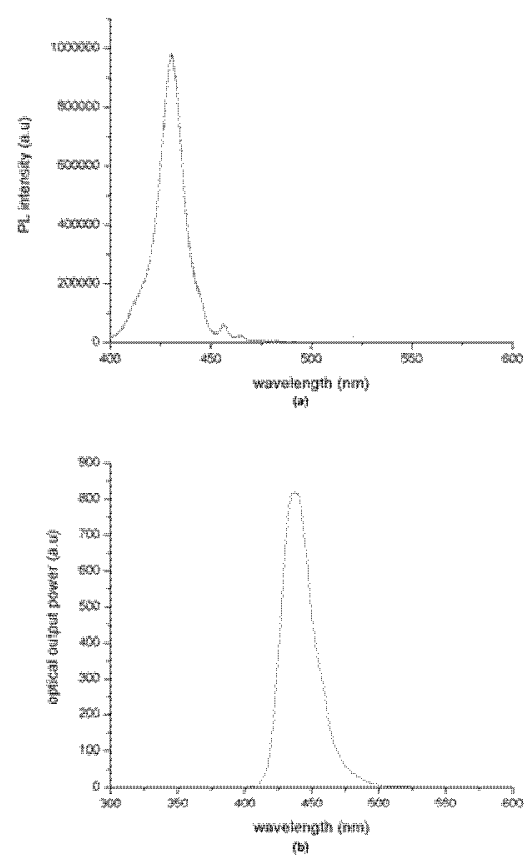

Figure 1. (a) Photoluminescense spectrum of the unprocessed epitaxial structure and (b) electroluminescense spectrum of the fabricated device.
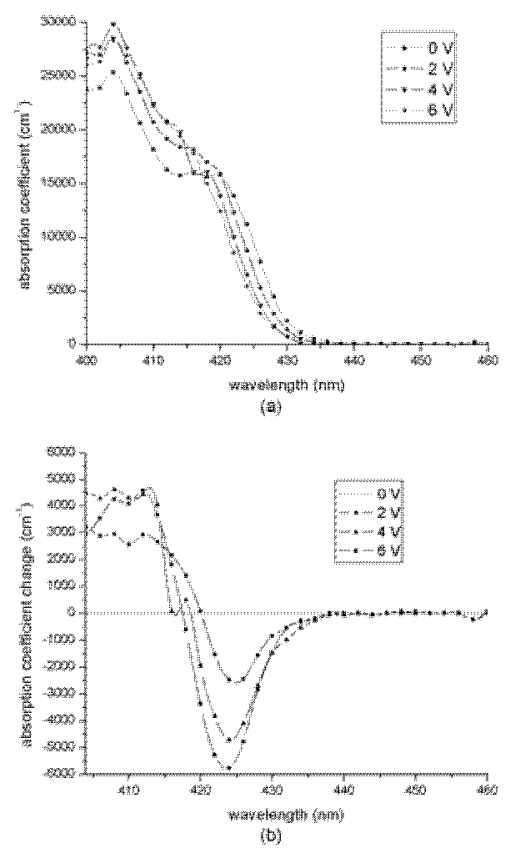

Figure 2. (a) Optical absorption spectra for various reverse bias voltages and (b) change in absorpton coefficient with respect to the absorption curve at $0 \mathrm{~V}$.
We electrically characterize $300 \mu \mathrm{mx} 300 \mu \mathrm{m}$ mesa devices using an HP4142 parameter analyzer. In forward bias, the inseries parasitic resistance is measured to be $<100 \Omega$. We also obtain the optical absorption spectra using photocurrent measurement setup that includes a Xenon lamp, a monochromator, a powermeter, a lock-in amplifier and a DC power supply for the application of various reverse bias voltages. Figure $2 \mathrm{a}$ shows the absorption spectra from $400 \mathrm{~nm}$ to $460 \mathrm{~nm}$ parameterized with respect to the reverse biases from $0 \mathrm{~V}$ to $6 \mathrm{~V}$. We observe an inflection point on the absorption curves at $418 \mathrm{~nm}$ for our InGaN/GaN quantum structures, which is in agreement with the work of Friel et al. on $\mathrm{AlGaN} / \mathrm{GaN}$ quantum structures [3]. This is an evidence of the polarization fields in $\mathrm{GaN}$ based quantum structures. Figure $2 \mathrm{~b}$ shows that electroabsorption change is maximum at $424 \mathrm{~nm}$ with a change of $\sim 6000 \mathrm{~cm}^{-1}$ with a $6 \mathrm{~V}$ swing (corresponding to $50 \mathrm{~cm}^{-1}$ absorption coefficient change for $1 \mathrm{~V} / \mu \mathrm{m}$ field change). This is a good operating wavelength with low background absorption and large absorption change as shown in Figs. $2 a$ and $2 b$.

We obtain the optical transmission spectra using an optical power meter and the same optical setup as in the photocurrent measurement. The transmission results agree with the photocurrent measurement including the same inflection point, although the stray light, not passing through the optical window of the modulator, renders a lower contrast (on/off) ratio in the transmission measurements.

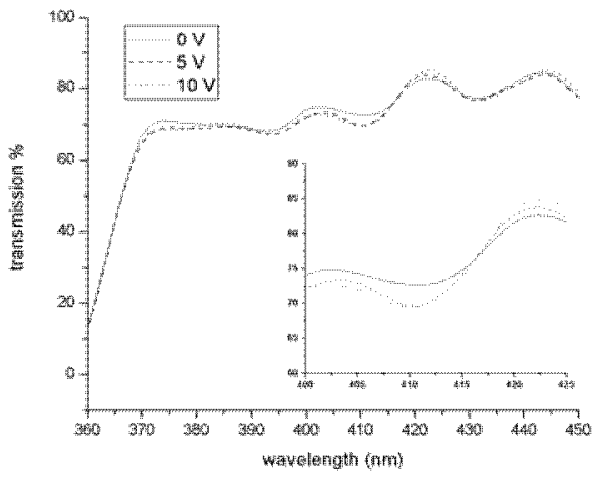

Figure 3. Transmission spectra for 0,5 and $10 \mathrm{~V}$ reverse bias, with the inset zoomed in around the inflection point.

\section{CONCLUSIONS}

We present blue InGaN/GaN-based quantum electroabsorption modulators for possible use in optical clock injection directly into Si CMOS in the blue. Unlike III-V modulators, these devices exhibit blue shift in their optical absorption with increasing external electric fields. 


\section{ACKNOWLEDGMENT}

This work is supported by a Marie Curie European Reintegration Grant MOON 021391 and EU-PHOREMOST Network of Excellence 511616 within the 6th European Community Framework Program and TUBITAK under the Project Nos. 104E114, 106E020, 105E065, and 105E066. H.V.D. and S.N. also acknowledge additional support from Turkish Academy of Sciences and TUBITAK.

\section{REFERENCES}

[1] A. Bhatnagar, et al., Journal of Lightwave Technology 22, No. 9, p.2213-17.

[2] S. Nakamura, et al., The Blue Laser Diode: The Complete Story, Springer, NY (2000).

[3] I. Friel, et al., J. Appl. Phys. 97, 123515 (2005). 